\title{
Follow-Up Imaging of Inflammatory Myofibroblastic Tumor of the Uterus and Its Spontaneous Regression
}

\author{
Biljana Markovic Vasiljkovic,, ${ }^{1, *}$ Vesna Plesinac Karapandzic, ${ }^{2}$ Tomislav Pejcic, ${ }^{3}$ Aleksandra \\ Djuric Stefanovic, ${ }^{1}$ Zorica Milosevic, ${ }^{2}$ and Snezana Plesinac ${ }^{4}$
}

${ }^{1}$ Center for Radiology and MRI, Clinical Center of Serbia, Medical Faculty, Belgrade University, Belgrade, Serbia

2 Institute of Oncology and Radiology, Medical Faculty, Belgrade University, Belgrade, Serbia

${ }^{3}$ Urological Clinic, Clinical Center of Serbia, Belgrade, Serbia

${ }^{4}$ Gynecological Clinic, Clinical Center of Serbia, Medical Faculty, Belgrade University, Belgrade, Serbia

${ }^{*}$ Corresponding author: Biljana Markovic Vasiljkovic, Center for Radiology and MRI, Clinical Center of Serbia, Medical Faculty, Belgrade University, Belgrade, Serbia. Tel: +38-163372956, Fax:+38-1112688553, E-mail: biljanamarkovicvasiljkovic@yahoo.com

Received 2013 July 12; Revised 2014 May 17; Accepted 2014 June 21.

\begin{abstract}
Inflammatory myofibroblastic tumor (IMT) is an aggressive benign mass that may arise from various tissues and organs with a great variability of histological and clinical appearances. Due to variable and nonspecific imaging findings, diagnosis of IMT is not obtained before surgery. The aim of this paper is to present CT and MRI findings during four-year follow-up of complete, spontaneous regression of IMT of the uterus. The diagnosis was made by histology and immunohistochemistry analysis of the open excisional biopsy specimen. At that time, the organ of origin was not specified. After analysis of the follow-up imaging findings and the mode of tumor regression, the uterus was proclaimed as the probable site of origin. IMT of the uterus is extremely rare and has been reported in ten cases up to now. The gradual, complete regression of uterine IMT documented by CT and MRI may contribute to understanding of its nature.
\end{abstract}

Keywords: Uterus, Inflammatory Pseudotumor, Neoplasm Regression, Spontaneous

\section{Introduction}

Inflammatory myofibroblastic tumor (IMT) is a rare benign lesion with a controversial etiology that mimics malignant neoplasm (1). It affects various tissues and organs (genitourinary tract, lung, and retroperitoneal space in different age groups (2). Clinical appearances of inflammatory pseudotumor (IPT) include inflammatory and reparatory processes, and myofibroblastic pseudosarcomatous proliferation (3). Inflammatory myofibroblastic tumor consists of spindle cells, acute and chronic inflammatory cells, and fibro-vascular stroma. Concerning predominant tissue component, IMT includes three histological subtypes (4). Histomorphology, ultrastructure and immunohistochemical analyses of IMT are complex when a variety of benign and malignant lesions has to be excluded $(3,5,6)$. The histological differentiation implies a few crucial points. First, detection of fibronexus and peripherally arranged contractile fibers by means of electron microscopy, positive immunostaining for smooth-muscle actin, desmin, calponin, fibronectin, some keratins and anaplastic lymphoma kinase (ALK) raise the possibility that spindle cells are truly myofibroblasts $(3,5)$. Second, benign myofibroblastic and fibroblastic lesions express ALK positivity with various expressions due to the anatomic site of the lesion while sarcomas do not. From the previously mentioned, leiomyosarcoma and fibrosarcoma might be separated from IMT $(1,3,4,7)$. Third, differentiation from idiopathic inflammatory lesions is based upon the higher percentage of cellularity and from the lymphoma by the presence of various clones of $\mathrm{T}$ and $\mathrm{B}$ lymphocytes $(3,5)$. A large amount of tissue specimen is necessary for such profound analysis and percutaneous biopsy is not advocated $(1,3)$.

Besides detection and evaluation of tumor extension, radiological presentation of IMT does not contribute to its characterization. Preoperative diagnosis of IMT refers to primary malignancy of the involving tissue or organ. Surgical excision is recommended whenever technically feasible (3). Spontaneous regression of abdominopelvic IMT is reported extremely rare in non-surgically treated patients (8-10). The gradual, complete regression of uterine IMT documented by computerized tomography (CT) and magnetic resonance imaging (MRI) may contribute to the understanding of its nature.

\section{Case Presentation}

A middle-aged female patient was admitted to the hos-

Copyright ( ) 2016, Tehran University of Medical Sciences and Iranian Society of Radiology. This is an open-access article distributed under the terms of the Creative Commons Attribution-NonCommercial 4.0 International License (http://creativecommons.org/licenses/by-nc/4.0/) which permits copy and redistribute the material just in noncommercial usages, provided the original work is properly cited. 
pital with a 5-month history of febrile condition, weight loss, left lumbar pain, and edema of the left leg. Spontaneous expulsion of the intrauterine device occurred 2 months before the onset of symptoms. Laboratory findings revealed an accelerated sedimentation rate, hypochromic anemia, leukocytosis, and a mild rise in serum creatinine level. Abdominal ultrasound and intravenous urography depicted bilateral hydronephrosis and a large, soft tissue mass occupying the pelvis. Bimanual vaginal and rectal examination confirmed pelvic mass involving the uterus, parametria, and mostly the left adnexa. Cystoscopy with biopsy did not show any abnormality and rectosigmoidoscopy showed extrinsic lumen narrowing with negative biopsy findings. A detailed clinical evaluation of this patient has been presented in the previous report (10). The first CT revealed an enlarged uterus with homogeneous wall thickening and indistinct outer and inner margins. The uterus was embedded within the tumor mass that extended toward the pelvic walls. After an intravenous contrast administration, the mass density raised heterogeneously (10 to $20 \mathrm{HU}$ ) without evidence of necrosis or calcification. Soft tissue planes between the uterus and bowels, ureters and both ovaries were erased (Figure 1). Explorative laparotomy was done. The tumor was of rubbery consistence tightly adherent to pelvic organs and the sacrum. Because a complete tumor excision was technically impossible, bowel liberation and excisional biopsy were performed. Routine hematoxylin-eosin (HE) stain showed hyalinized stroma with myofibroblasts and numerous inflammatory cell infiltrates (Figure 2). Immunohistochemical analysis verified spindle cell positivity for actin and vimentin, inflammatory cell positivity for LCA and CD68, and vascular vessel wall positivity for anti-CD34 antibody. Immunohistochemical findings were negative for desmin, S-100, cytokeratin HMV, and focally positive for cytokeratin MNF116. The pathological diagnosis was inflammatory myofibroblastic tumor of unknown origin. Meanwhile, the patient underwent unilateral (right) percutaneous nephrostomy (PCN) catheter insertion. Broad-spectrum antibiotics were administered during a one-month period. Inflammatory symptoms and laboratory findings returned to normal within three months when PCN was extracted. Then, 4 months after surgery, the second CT depicted reduction of the tumor volume while its densities on both unenhanced and enhanced scans remained identical with the first CT. Eight months after the initial work-up, further tumor regression was noted with iso- and hypodense strands of tumor tissue arranged in the radial fashion connecting uterus and pelvic walls (Figure 3). Further on, ultrasound checkups were done periodically every 6 months, in which uterus wall thickening and its indistinct outer border were present. Two years after the initial diagnosis, follow-up CT depicted complete tumor regression with restoration of pelvic organ morphology and involution of the uterus to dimensions appropriate to the patient's age. The only apparent abnormality was an invisible fat tissue plane between the uterus and the adjacent bowel loops, thus suggesting adhesions (Figure 4). MRI exams were done during the third, fourth and fifth year of follow-up. In T1 weighted fat suppression sequence, T2 weighted fat suppression sequence, fast spin echo sequence, pelvic organs with normal topography and morphology without residual periuterine fibrosis were depicted. The uterus corpus, cervix zone anatomy, and the width of the endometrium were within normal limits (Figure 5). Diffusion weighted images were obtained during these MRI exams and no apparent changes were observed (Figure 6).
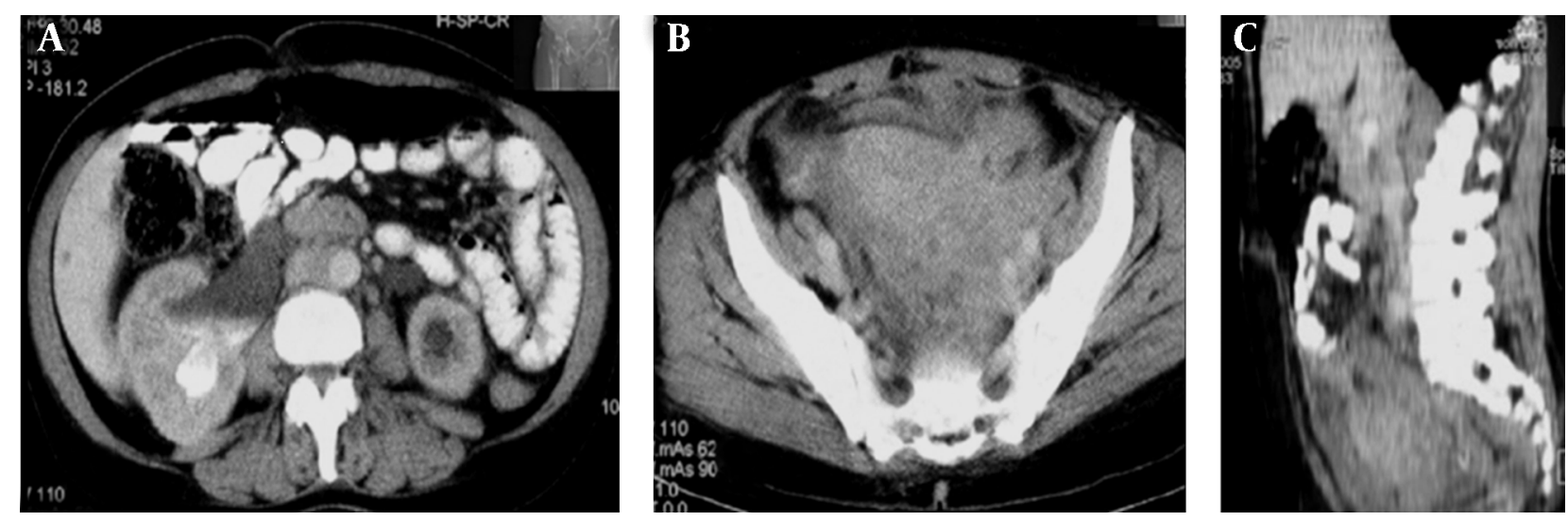

Figure 1. A middle-aged female with a 5-month history of febrile condition, weight loss, left lumbar pain, and edema of the left leg; A and B, Axial images of the initial abdominopelvic CT, right kidney hydronephrosis; C, Sagittal reformatted image during the late parenchymal phase. Inflammatory myofibroblastic tumor (IMT) occupies the entire pelvis. It is presented as a soft tissue mass, hypodense to skeletal muscles and uterus. Fat planes between pelvic organs and sacrum are erased due to infiltrative tumor growth. 


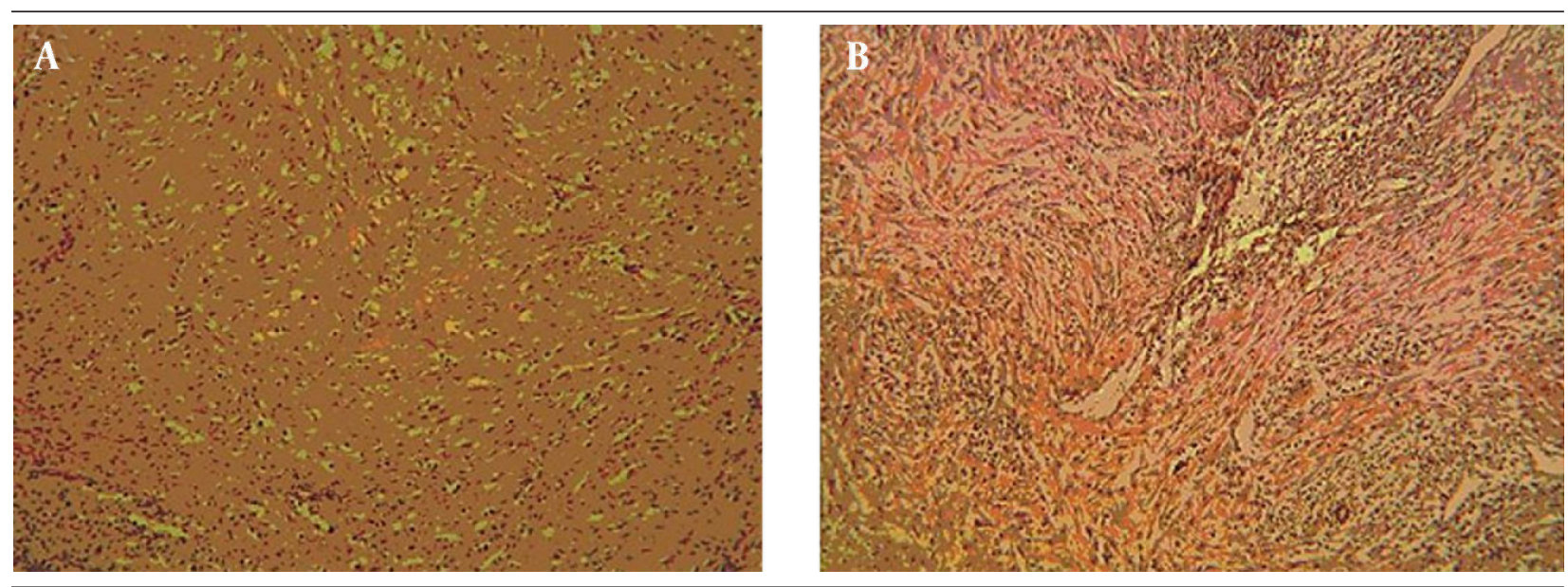

Figure 2. Histologic specimens. A, Myofibroblastic proliferation with dense hyalinization and inflammatory infiltrate (Hematoxylin-eosin staining with 100 times magnification $(\mathrm{H} \& \mathrm{E}, \times 100)$ ); B, Myofibroblastic proliferation with dense lymphoplasmocytic infiltration (H\&E, $\times 100)$. Mitoses and atypia of the cells were not observed.
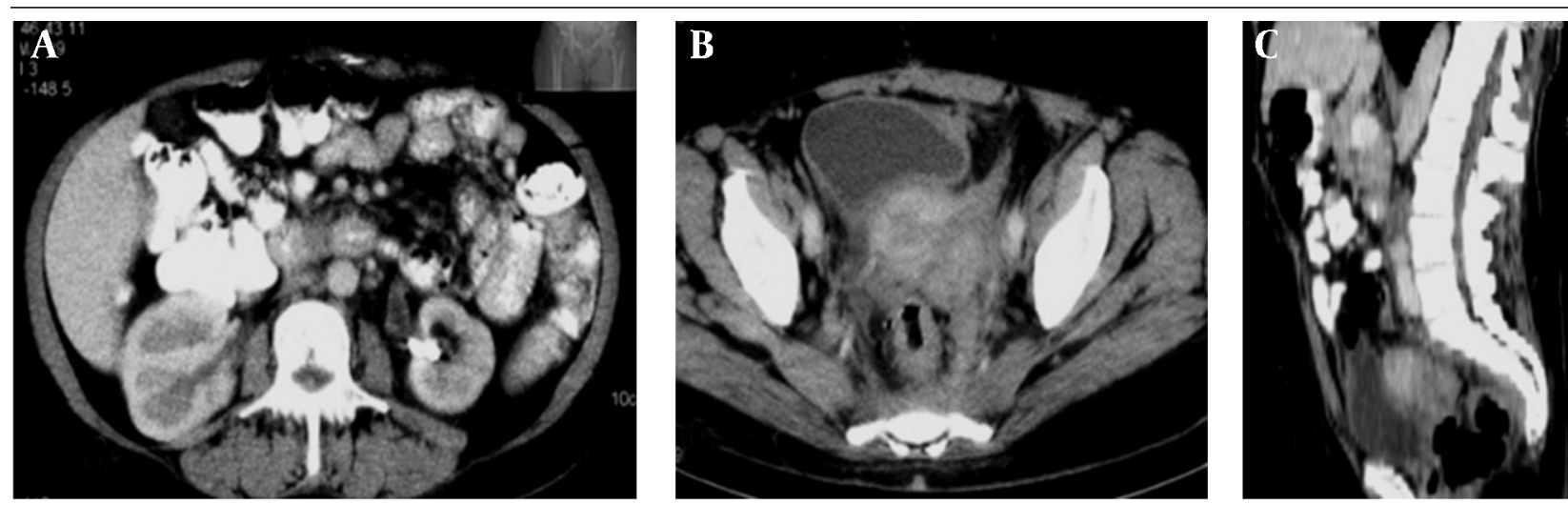

Figure 3. CT scan eight months after the initial work up. A and B, Axial images; C, Sagittal reformatted image during the late parenchymal phase. Both kidneys restored normal morphology. Proximal part of the left ureter is still slightly dilated. Reduction in IMT tumor volume is apparent. Uterine cavity can be recognized. Soft tissue bands, slightly hypodense to the uterus, traverse perirectal, perivesical, and pre-sacral space are seen.
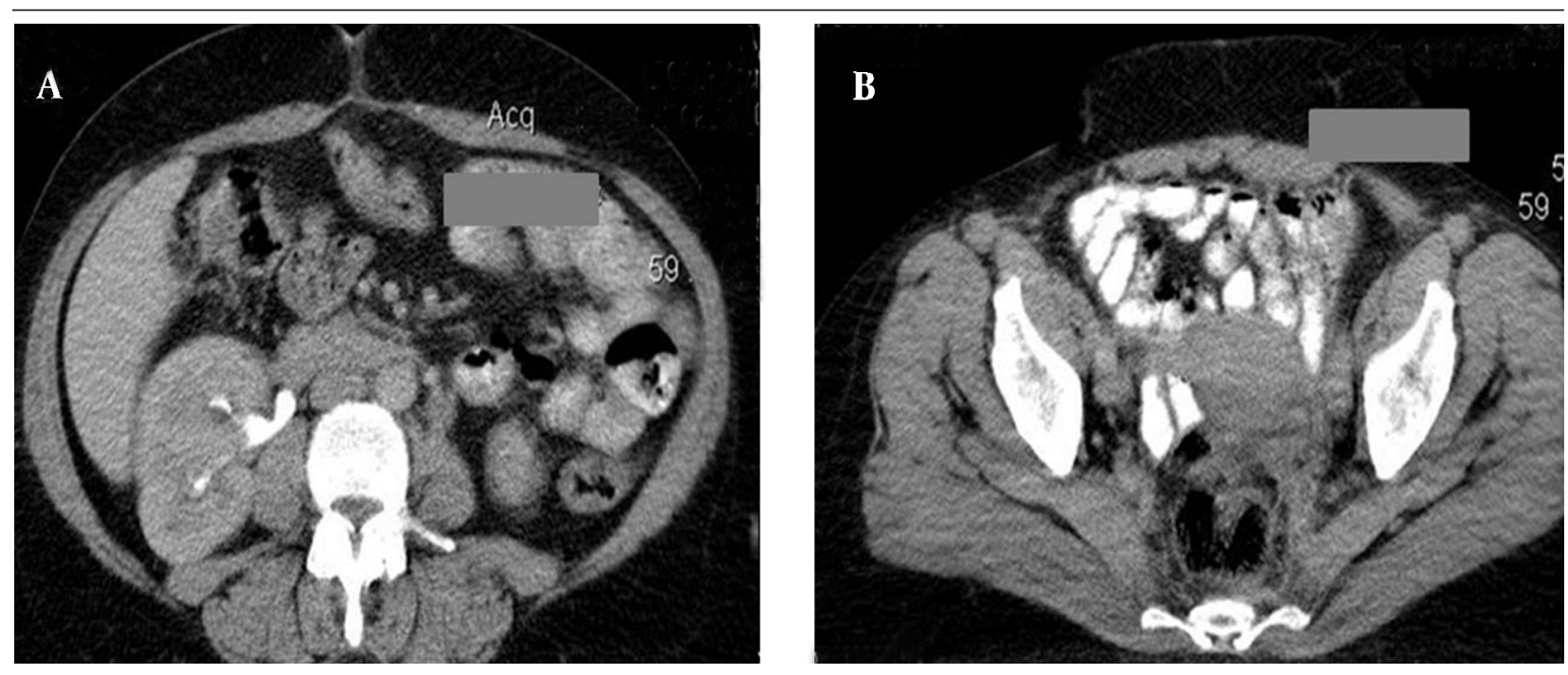

Figure 4. CT scan 24 months after the initial work up. A, Axial image at the level of the midabdomen; B, Axial image at the level of the uterine body during the excretory phase. Complete regression of the pelvic inflammatory myofibroblastic tumor is apparent with recuperation of the right kidney hydronephrosis (left kidney not shown). The outer margins of the uterus are indistinct from the bowel loops suggesting adhesions. 

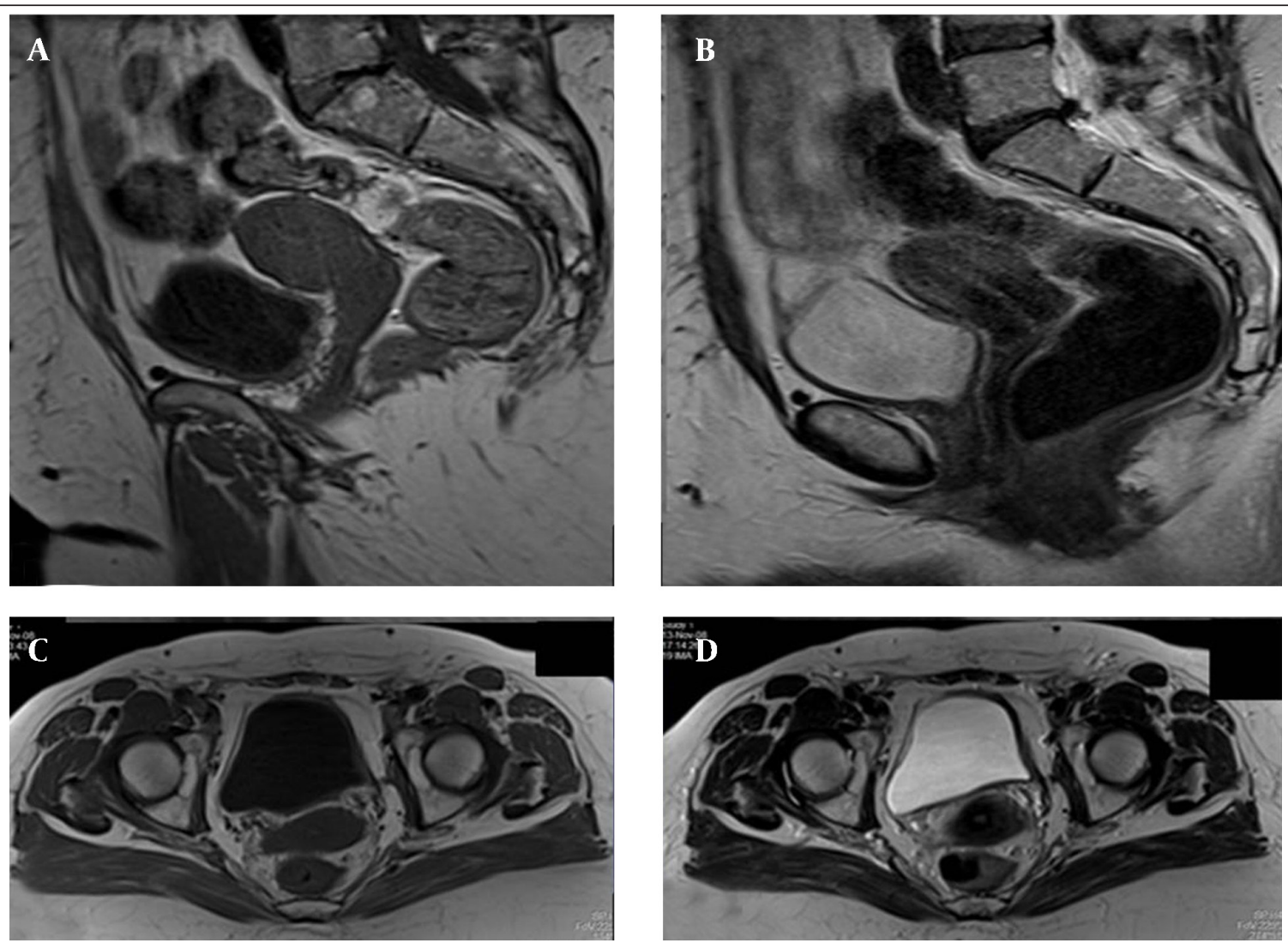

Figure 5. MRI of the pelvis four years after the initial work up. A, Sagittal T1 weighted fat suppressed sequence(T1W FS); B, Axial T1W FS sequences; C, Sagittal T2W FS; D, Axial T2W FS sequences. Uterus and vagina morphology is completely normal according to the patient's age. Uterus is slightly distracted to the left pelvic wall. Anatomical zone of the uterus is preserved and the endometrium has a normal width. Parametria is normal too.
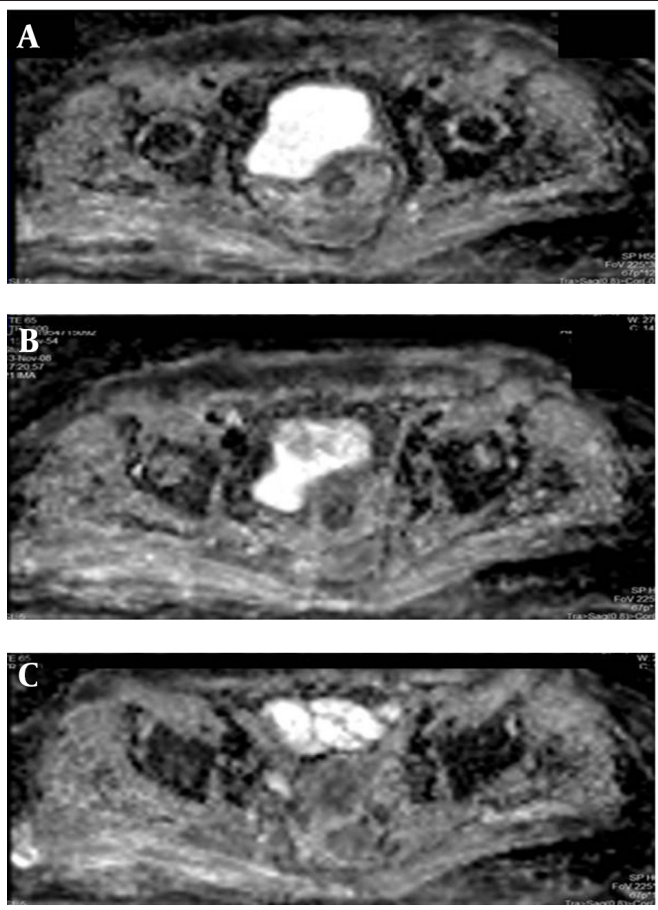

Figure 6. Diffusion weighted images of the pelvis on the 5-year follow-up MRI. There is no evidence of qualitative changes of water diffusion in the region of interest.

\section{Discussion}

The majority of published reports refer IMT under several names such as IPT (inflammatory pseudotumor) or inflammatory myofibrohistiocytic proliferation, but the histological entity is the same (3). The real incidence of IMT is unknown and the proper evaluation may often be doubtful (2). Reports on IMT (inflammatory pseudotumor) of the uterus in 11 female patients, aged 6 to 63, have been published (11-13). Clinically, these cases presented themselves with constitutional symptoms, symptoms due to the local tumor growth and its mass effect, while some were asymptomatic. In all the published reports except one, tumors were surgically excised and the diagnosis was made by histological and immunohistochemical study. Spindle cells contained in IMT were positive on smooth muscle actin in all cases. In recent reports, published after 2003, the positivity on ALK was referred to $(11,12)$. Two types of growth pattern were observed: intracavitary- polypoid (five patients) and extracavitary- infiltrative (six patients). Localized, polypoid form of uterine IMT did not invade the endometrium and was indistinguishable from leyomyoma by means of imaging (12). The case of uterine IMT in which decrease and normalization of the serum level of IL-6 occurred after surgical excision emphasized the inflammatory component of IMT (13). 
In these reports, apart from detection and staging, little attention has been paid to the imaging presentation of uterine IMT. Concerning its imaging characterization, the general opinion is that IMT mimics other malignancies. Therefore, very few papers have been published about the radiological presentation of abdominopelvic IMT $(8,14)$. Polypoid form of IMT is referred to other hollow structures such as the bowels, ureter and urinary bladder $(8,14,15)$. Calcium deposits are observed in liver, pelvic, pancreatic and stomach IMT (IPT). IMT on noncontrast CT scans can be isodense or hypodense when compared to skeletal muscles. The necrosis is presumed possible in large volume IMT. After contrast administration, CT density can vary. It can be homogeneous, heterogeneous, early peripheral or late central (14). MRI presentation of IMT also varies significantly. A majority of cases of IMT were isointense and hypointense in T1W sequences and hyperintense in $\mathrm{T} 2 \mathrm{~W}$ sequences compared to the surrounding muscles. The different degree of hyperintensity in $\mathrm{T} 2 \mathrm{~W}$ sequences correlates with the amount of fibrotic component revealed histologically. Some authors imply that an increase in tumor hyperintensity in $\mathrm{T} 2 \mathrm{~W}$ sequences stresses tumor evolution toward sclerosis and implicates good prognosis. Partial tumor regression refers to a situation in which intensity parameters remain the same on the follow-up MRI (9). After contrast administration, IMT shows heterogeneous increase in signal intensity thus making it impossible to differentiate it from various malignant lesions (9). The contrast enhancement (CT, MRI) depends on vascularization of the lesion and in case of IMT, vascular pattern depends on predominant histological subtype and it does not correlate with benign/malignant nature, tumor extension or its prognosis.

During the first two-year period of follow-up, we used ultrasound and CT imaging in order to obtain more anatomical details. Further on, we preferred MRI imaging to avoid unnecessary radiation and to achieve better tissue differentiation.

In IMT, the word "inflammatory" defines diffuse tumor infiltration with cells of acute and chronic inflammation. A causative, infective agent is detected in IMT of the spleen, lymph nodes (Epstein Barr virus) and in some cases of lung IMT (mycoplasma) (6). Success with steroid treatment in cases of IMT of the lung and resolved constitutional symptoms with normalization of IL-6, and IL-1 serum levels after surgery implicate inflammatory etiology of some IMTs $(12,13)$. In our case, complete resolution of clinical signs and laboratory abnormalities were accompanied by a decrease in tumor volume. However, the tumor mass was still significant at that time. Restored kidney function remained stable after extraction of PCN. This indicates that although still present, tumor does not compress ureters significantly (second CT). Normal morphology of the pelvicaliceal system of both kidneys and normal serum creatinine level persistent after 4-year follow-ups are indirect signs that tissue restoration was complete and with no evidence of ureteral or periureter- al fibrosis. During tumor regression, no sign of necrosis or abscess formation was evident on CT exams. We noted an interesting mode of tumor regression. Tumor volume decreased gradually from the periphery toward the uterus, while the enhancement pattern (slightly higher at the outer tumor margins) remained unchanged. Restoration of the pelvic organ morphology in such a manner is called "rewind backwards". It is similar to any inflammation extension, only in the opposite direction. At the end of follow up no residual mass or dystrophic calcifications were present. Both findings were previously reported (9). This report of complete IMT regression contributes to the benign, immunologically mediated nature of some IMTs. However, all authors agree that thorough follow-up is mandatory in all cases of IMT (IPT). This attitude is supported with cases of IMT recurrence years after the surgery as well as with secondary malignancies that were either undetectable or the IMT was improperly diagnosed from the beginning $(2,7,8)$.

Controversial etiology including trauma, infection, and malignancy, and no specific immunohistochemical and genetic abnormalities implicate that IMT is the diagnosis of exclusion $(3,5,6)$. Radiological and histological findings have to be complemented with the clinical parameters.

Radiological presentation of IMT does not enable proper diagnosis before surgery. In cases in which the data suggest that previous trauma and constitutional symptoms are present, IMT should be encountered in the differential diagnosis of the pelvic mass. Absence of necrosis, the "rewinding backwards" type of regression without residual fibrosis emphasize the fact that IMT was of benign nature, and that the uterus is the site of origin. This is the first radiologically documented follow-up of gradual and complete regression of uterine IMT.

\section{Footnotes}

Authors' Contribution:All the authors substantially contributed the manuscript preparation, read the final paper version and approved it.

Financial Disclosure:All the authors state that there are no financial or other conflicts of interests.

Funding/Support:There was no financial support.

\section{References}

1. Coffin CM, Fletcher JA. Inflammatory myofibroblastic tumor. In: Fletcher CDM, Unni KK, Mertens F, editors. World Health Organization classification of tumours. Pathology and genetics of tumours of soft tissue and bone. Lyon: IARC Press; 2002. pp. 91-3.

2. Janik JS, Janik JP, Lovell MA, Hendrickson RJ, Bensard DD, Greffe BS. Recurrent inflammatory pseudotumors in children.J Pediatr Surg. 2003;38(10):1491-5. [PubMed: 14577073]

3. Gleason BC, Hornick JL. Inflammatory myofibroblastic tumours: where are we now? J Clin Pathol. 2008;61(4):428-37. doi: 10.1136| jcp.2007.049387. [PubMed: 17938159]

4. Coffin CM, Watterson J, Priest JR, Dehner LP. Extrapulmonary inflammatory myofibroblastic tumor (inflammatory pseudotumor). A clinicopathologic and immunohistochemical study of 84 cases. Am J Surg Pathol. 1995;19(8):859-72. [PubMed: 7611533] 


\section{Markovic Vasiljkovic B et al.}

5. Eyden B, Banerjee SS, Shenjere P, Fisher C. The myofibroblast and its tumours. J Clin Pathol. 2009;62(3):236-49. doi: 10.1136 jcp.2008.061630. [PubMed: 18930983]

6. Dehner LP. The enigmatic inflammatorypseudotumours: the current state of our understanding, or misunderstanding. J Pathol. 2000;192(3):277-9. doi: 10.1002/1096-9896(200011)192:3<277::aidpath749>3.0.co;2-e. [PubMed:11054708]

7. Coffin CM, Hornick JL, Fletcher CD. Inflammatory myofibroblastic tumor: comparison of clinicopathologic, histologic, and immunohistochemical features including ALK expression in atypical and aggressive cases. Am J Surg Pathol. 2007;31(4):509-20. doi: 10.1097/01.pas.0000213393.57322.c7. [PubMed:17414097]

8. Jimenez JM, Poustchi-Amin M, Leonidas JC, Pena A. Extraperitoneal abdominopelvic inflammatory pseudotumor: report of four cases. Pediatr Radiol. 1997;27(2):170-4. doi: 10.1007| s002470050093. [PubMed: 9028854]

9. Venkataraman S, Semelka RC, Braga L, Danet IM, Woosley JT. Inflammatory myofibroblastic tumor of the hepatobiliary system: report of MR imaging appearance in four patients. Radiology. 2003;227(3):758-63. doi: 10.1148/radiol.2273020572. [PubMed: 12728186]

10. Plesinac-Karapandzic V, Perisic Z, Milovanovic Z, Vukicevic D,
Mileusnic D, Stevanovic J, et al. Invasive inflammatory pseudotumor of the pelvis: a case report with review of the literature. $J$ BUON. 2009;14(2):301-6. [PubMed:19650182]

11. Shintaku M, Fukushima A. Inflammatory myofibroblastic tumor of the uterus with prominent myxoid change. Pathol Int 2006;56(10):625-8. doi:10.1111/j.1440-1827.2006.02018.x. [PubMed: 16984620]

12. Rabban JT, Zaloudek CJ, Shekitka KM, Tavassoli FA. Inflammatory myofibroblastic tumor of the uterus: a clinicopathologic study of 6 cases emphasizing distinction from aggressive mesenchymal tumors. Am J Surg Pathol. 2005;29(10):1348-55. [PubMed: 16160478]

13. Azuno Y, Yaga K, Suehiro Y, Ariyama S, Oga A. Inflammatory myoblastic tumor of the uterus and interleukin-6. Am J Obstet Gynecol. 2003;189(3):890-1. [PubMed: 14526339]

14. Narla LD, Newman B, Spottswood SS, Narla S, Kolli R. Inflammatory pseudotumor. Radiographics. 2003;23(3):719-29. doi: 10.1148/ rg.233025073. [PubMed: 12740472]

15. Park SB, Cho KS, Kim JK, Lee JH, Jeong AK, Kwon WJ, et al. Inflammatory pseudotumor (myoblastic tumor) of the genitourinary tract. AJR Am J Roentgenol. 2008;191(4):1255-62. doi: 10.2214/ AJR.07.3663. [PubMed: 18806173] 